\title{
Regular Alternation of Fiber Types in the Transversus Abdominis Muscle of the Garter Snake ${ }^{1}$
}

\author{
ROBERT S. WILKINSON AND JEFF W. LICHTMAN \\ Department of Physiology and Biophysics, Washington University School of Medicine, St. Louis, Missouri 63110
}

\begin{abstract}
The snake transversus abdominis muscle is an extremely simple segmentally repeating muscle containing 80 to 100 muscle fibers in a single-fiber-thick sheet. This muscle exhibits a striking pattern of muscle fiber types: twitch fibers alternate with tonic fibers and, among the twitch fibers, slower and faster contracting subtypes also alternate. Thus, in many regions of the muscle the pattern of fiber types is: faster twitch, tonic, slower twitch, tonic, faster twitch, tonic, and so on. The existence of a spatial pattern of fiber types, perhaps discernible in this muscle because of the muscle's extreme geometrical simplicity, provides good evidence for an intrinsic component to muscle fiber differentiation.
\end{abstract}

Vertebrate muscles typically contain a variety of muscle cell phenotypes. These fiber types differ in contractile and metabolic properties as well as in their source of innervation. Although this cellular diversity is obvious in adult animals, its origin during development is not understood. There are two principal ways in which muscle fiber type heterogeneity could arise: intrinsic differences among muscle fiber precursors might give rise to the different adult fiber types (Nougues and Bacou, 1977; Butler et al., 1982; Thompson et al., 1984), or motor axons might dictate the properties of the fibers they innervate (Askansas et al., 1972; Rubinstein and Kelly, 1978). Because there is evidence supporting both intrinsic and neural influences as the primary cause of muscle fiber differentiation, the relative importance of these two factors has not been resolved (for review see Kelly, 1983). One difficulty in approaching this question directly has been that adult muscles show little evidence of the developmental strategy which produced their fiber types. Indeed, at the cellular level, fibers of different types seem to be arranged in an apparently random "mosaic" pattern (Karpati and Engel, 1968). Here we describe an unusual muscle which displays a predictable cellular pattern of fiber types. This stereotyped pattern provides a unique opportunity to examine at the cellular level how developmental influences determine the adult phenotype.

\section{Materials and Methods}

Adult garter snakes were cold-anesthetized in ice water ( $15 \mathrm{~min}$ ) and killed by decapitation. Sections of snake 2 to $3 \mathrm{~cm}$ in length were cut, eviscerated, and placed in refrigerated reptilian saline solution (composition in millimolar concentration: $\mathrm{NaCl}, 145 ; \mathrm{KCl}, 2.5 ; \mathrm{CaCl}_{2}, 3.6 ; \mathrm{MgSO}_{4}, 1.8 ; \mathrm{KH}_{2} \mathrm{PO}_{4}, 1.0$;

Received January 31, 1985; Revised April 23, 1985;

Accepted May 6, 1985

1 We thank D. Dill for technical assistance, and D. Purves and J. Sanes for critical comments. This work was supported by National Institutes of Health Grant NS20364 and by the Muscular Dystrophy Assocation. J. W. L. is a Sloan Fellow.
HEPES, 5.0; glucose 3.0; $\mathrm{NaOH}$ as needed for $\mathrm{pH} 7.4$ ). Snake sections could be stored in saline for several days with no apparent deterioration.

The transversus abdominis is a thin segmental muscle coursing between each rib and the ventral midline. Several contiguous segmental components of this and the overlying external oblique (see Kuffler and Yoshikami, 1975) muscles were exposed from the ventral side and removed along with their origins on the ribs and midline insertions at the linea alba. For a particular segmental component the overlying external oblique muscle was then carefully dissected away to reveal the transversus abdominis muscle and its nerve (see Fig. 1). Generally more rostral segments were preferred as the underlying connective tissue was relatively free of pigmentation, permitting better visualization of the muscle fibers.

Electrophysiological experiments were performed at room temperature $\left(25^{\circ} \mathrm{C}\right)$ on the stage of an inverted Nomarski microscope. Intracellular microelectrodes were filled with $2 \mathrm{M}$ potassium acetate (resistance, 80 to 120 megohms). Possible damage to fibers due to impalement was assessed by noting the change in resting potential recorded by one microelectrode due to impalement by a second. Resting potentials typically decreased by $5 \mathrm{mV}$ or less and then returned to their original values over a period of $\sim 10$ to 30 sec.

Passive electrical properties of muscle fibers were studied using two microelectrodes, one for the passage of current, the other for recording of membrane potential change. Electrodes were spaced $50 \mu \mathrm{m}$ apart, using a calibrated reticle in one of the microscope's eyepieces. This distance was sufficient to reduce stimulus artifacts in the recording electrode to a satisfactory level, yet was negligible compared to the electronic length constant of the fibers, the latter being estimated from amplitude and decay times of miniature endplate potentials recorded at various distances from the endplate (see also Lichtman et al., 1985)

Input resistance $\left(R_{\text {in }}\right)$ and an effective membrane time constant $\left(\tau_{\mathrm{m}}\right)$ were measured using hyperpolarizing square current pulses (amplitude, 2 to 50 $\mathrm{nA}$; duration, 20 to $200 \mathrm{msec}$ ) sufficient to cause membrane potential changes of approximately 10 to $20 \mathrm{mV}$. $R_{\text {in }}$ was taken as the ratio of steady state voltage to current, and $\tau_{m}$ was taken as that time required for the membrane potential transition to reach $84 \%$ of its steady-state value, in accord with infinite cable theory where the distance between recording and stimulation sitcs is ncgligible (Hodgkin and Rushton, 1946). In some moas urements of $R_{\text {in }}$ and $\tau_{\mathrm{m}}$ (20 fibers) and in all tension measurements, a minicomputer (model 980B, Texas Instruments) was used for the generation of stimuli and for the acquisition of membrane potential and tension records (resolution of analogue-to-digital converters, 1 part in 4096; sampling frequency, $500 / \mathrm{sec}$ to $5000 / \mathrm{sec}$ ). In these measurements, digitized records of membrane voltage responses to current pulses were displayed on a cathode ray tube (CRT) together with the appropriate theoretical error function curve (Hodgkin and Rushton, 1946); $\tau_{\mathrm{m}}$ was obtained by adjusting the time base of the theoretical curve for best fit to the data. This procedure confirmed that both tonic and twitch fibers behaved as infinite cables for purposes of measuring $\tau_{\mathrm{m}}$.

Tension of individual fibers was measured using a commercial tension transducer (model 407, Cambridge Technology) whose amplified output fed an analogue-to-digital converter. Identical single twitches could be easily elicited either by extracellular focal stimulation near an endplate ( $200 \mu \mathrm{sec}$, 10- to $50-\mathrm{V}$ depolarizing pulses; amplitude below threshold for activating the innervating axon) or by intracellular current pulses (see Fig. 7 legend). Tetanic contractions were more difficult to elicit with extracellular stimulation: voltages 
required to stimulate small fibers away from their endplates were invariably quite high and often exceeded the threshold of adjacent larger fibers, thus contaminating tension records. Stimulation adjacent to endplates was also unsatisfactory because the endplates moved slightly with respect to the stimulus pipette as tetanic contraction was initiated (even though overall contraction was isometric), making it necessary to "track" the endplate manually with the pipette. For these reasons intracellular stimulation was preferred. Tension records obtained by intracellular stimulation were deemed acceptable only when resting potential remained unchanged after a train of stimuli was delivered, and when several stimulus trains delivered in succession produced identical tetanic tension records.

\section{Results}

The transversus abdominis of the garter snake comprises approximately 200 segmental components per side, each of which extends ventromedially from a rib to a tendonous sheath (linea alba) at the ventral midline (Fig. 1). A unique feature of the transversus abdominis is that the $\sim 80$ fibers within each segmental component fan out upon leaving the rib into a single-fiber-thick sheet. Because of this extreme thinness, all muscle fibers and nerve-muscle contacts are readily visible in both living and whole mounted fixed preparations.

Each segmental component is provided with innervation from the corresponding ventral segmental nerve which gives off a transversus abdominis muscle branch before continuing ventromedially as a large cutaneous nerve trunk. Once the muscle nerve enters the plane of the muscle it branches to supply one main and several smaller endplate bands which run approximately perpendicular to the long axis of the fibers. Often, a few fibers at the edge of an adjoining segment are supplied as well.

Electrophysiological studies using two intracellular electrodes (see "Materials and Methods") indicate two discrete, nonoverlapping classes of fibers within this muscle. About half of the muscle fibers have low input resistance, $R_{\text {in }}$ (range, 0.4 to 1 megohm; $N=25$ ), short membrane time constants $\tau_{\mathrm{m}}$ (range, 4 to $8 \mathrm{msec} ; N=25$ ), and always generate overshooting action potentials when depolarized by intracellular current pulses ( $2 \mathrm{msec}, 20$ to $50 \mathrm{nA}$ ) or by nerve stimulation (Lichtman et al., 1985). Action potentials are accompanied by a brisk twitch. The second major class contains muscle fibers with higher $R_{\text {in }}$ (range 1.5 to 6 megohms; $N=25$ ) and longer $\tau_{\mathrm{m}}$ (range 10 to $28 \mathrm{msec} ; N=25$ ). These fibers lack any regenerative response to injected current, even when depolarized beyond +100 $\mathrm{mV}$, and they do not fire action potentials when their innervating nerve is stimulated (Lichtman et al., 1985). They produce slow, tonic, focal contractions in proportion to the magnitude of depolarization. Diameters of these tonically contracting fibers are, on average, smaller than those of the twitch fibers, although the latter are quite variable in size, often being larger near the edges than near the center of the muscle.

The type of innervation supplying the two principal fiber types is also distinct. Gold chloride staining (Boyd, 1962) reveals that each twitch fiber is innervated by a solitary large terminal containing $\sim 60$ boutons (Fig. 2a) in the main endplate band located about midway along the muscle's length. In contrast, each tonic fiber is innervated at six to eight smaller, elongated endplates, each containing $\sim 17$ boutons (Fig. $2 \mathrm{~b}$ ) spaced at approximately equal $1-\mathrm{mm}$ intervals along its length. The shape of these endplates is much narrower than that of the endplates on twitch fibers (Table I), and they are often multiply innervated (Lichtman et al., 1985). Both types of terminals are visible in the main endplate band (Fig. 3a), but only the smaller terminals are present in the exclusively tonic fiberinnervating endplate bands (Fig. $3, b$ to $d$ ). Table I lists the major differences between the two principal fiber types.

In many regions of the muscle, twitch and tonic fiber types alternate to give a stereotyped pattern. The alternation is often discerned easily as a sequence of large and small fiber diameters (Fig. 3 ) but is more reliably assessed by making use of the clear morphological differences in innervation of the two fiber types (Figs. 2 and 3 ) or by electrophysiological recordings (Table I). Thus, in the main endplate band, which contains both twitch and tonic muscle fiber innervation, an alternation of large and small terminals is often obvious (Fig. 4, see also Fig. 3a). Consistent with this, within any of the endplate bands that innervate tonic but not twitch fibers, only every second fiber is innervated (Fig. $3, b$ to $d$ ).

To assess the fidelity of the alternating pattern, the criteria of terminal size (number of boutons and width) and number of terminals per fiber (Table I) were used to classify each of the fibers in eight contiguous segments of the transversus abdominis stained with gold chloride. The pattern of fiber types among 669 consecutive fibers identified by these morphological criteria is shown in Figure 5 . In

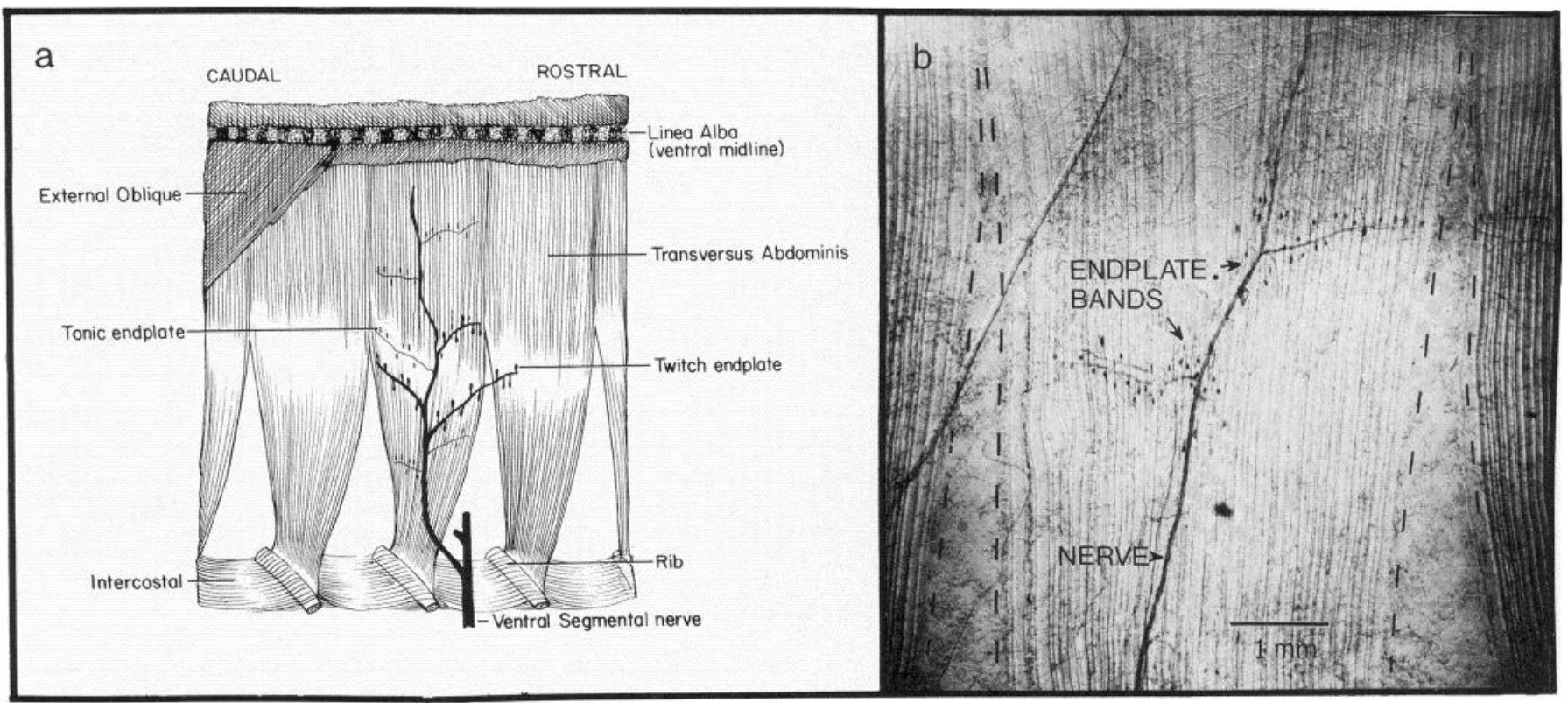

Figure 1. a, Diagrammatic representation of the snake transversus abdominis muscle. Each segmental muscle originates on a separate rib (bottom) and courses ventromedially to the midline (above) where it inserts beneath the external oblique muscle. Each component ( $\sim 80$ fibers) contains approximately equal numbers of twitch and tonic fiber types; endplate bands run roughly perpendicular to the long axis of the fibers. $b$, Low power photomicrograph (gold chloride stain) corresponding to the central region in a. Segmental components are shown separated by dashed lines. 

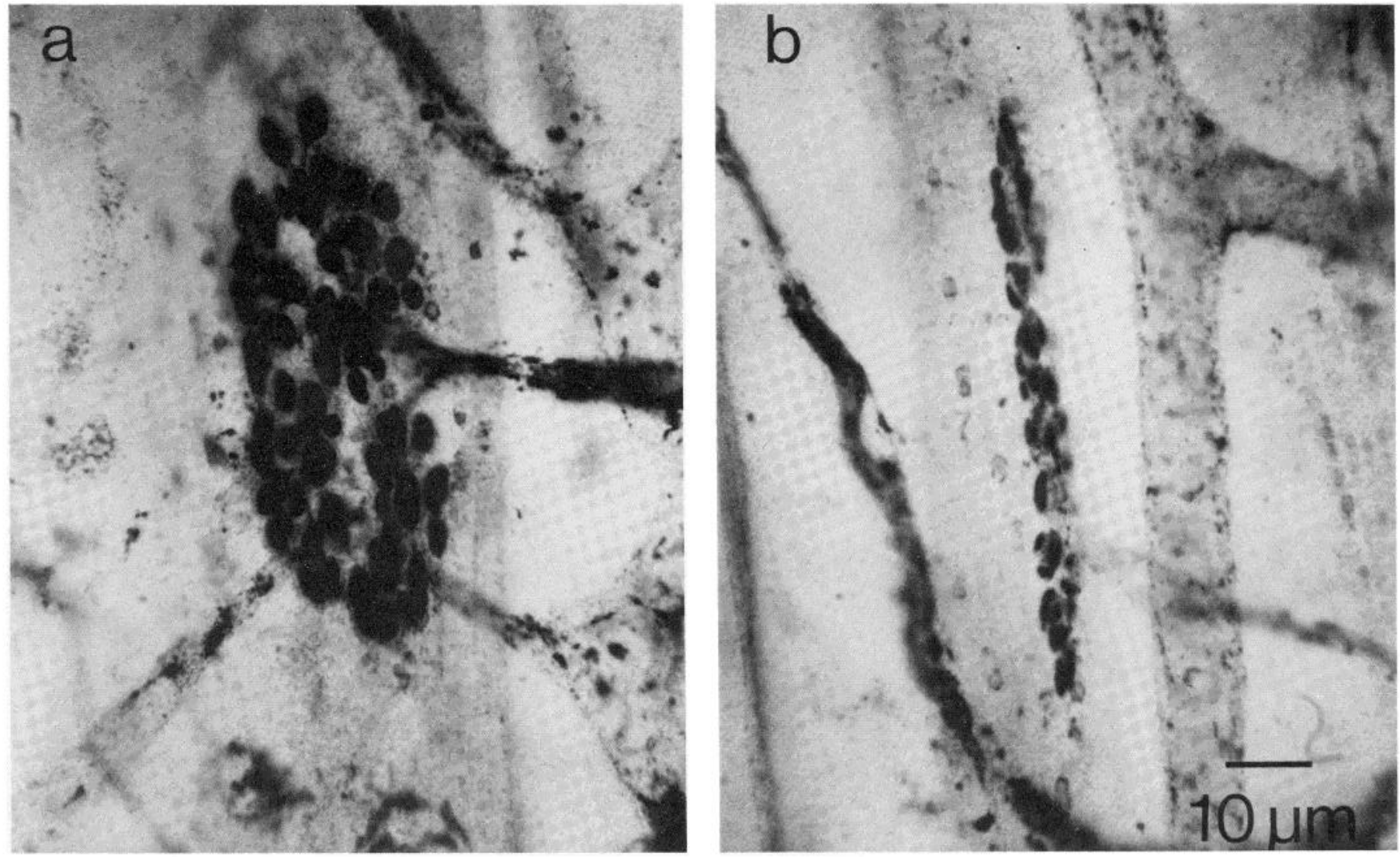

Figure 2. Photomicrographs of twitch (a) and tonic (b) nerve terminals in the transversus abdominis. Gold chloride stain. Tonic terminals contain fewer boutons and are more elongated than terminals innervating twitch fibers (see also Cole, 1955).

TABLE I

Properties of twitch and tonic muscle fibers in the snake transversus abdominus muscle

\begin{tabular}{lcc}
\hline & Twitch $(N=25)$ & Tonic $(N=25)$ \\
\hline Action potential & yes & $\mathrm{no}^{a}$ \\
Diameter $(\mu \mathrm{m})$ & $50 \pm 21^{b}$ & $26 \pm 6$ \\
Endplate length $(\mu \mathrm{m})$ & $50 \pm 10$ & $41 \pm 9$ \\
Endplate width $(\mu \mathrm{m})$ & $23 \pm 5$ & $8 \pm 2$ \\
Number of endplates/muscle fiber & $1 \pm 0$ & $7.3^{c}$ \\
Number of axons/endplate ${ }^{\sigma}$ & $1 \pm 0$ & $1.6 \pm 0.6$ \\
Number of boutons/endplate & $58 \pm 15$ & $17 \pm 6$ \\
Resting potential $(\mathrm{mV})$ & $-74 \pm 6$ & $-59 \pm 13$ \\
$R_{\text {in }}$ (megohms) & $0.63 \pm 0.27$ & $3.2 \pm 1.2$ \\
$\tau_{\mathrm{m}}$ (msec) & $5.1 \pm 1.1$ & $21 \pm 4$ \\
\hline
\end{tabular}

${ }^{a}$ Tonic fibers showed no sign of regenerative response to depolarization. Both fiber types showed voltage- and time-dependent rectification.

${ }^{b}$ Data presented are mean values $\pm \mathrm{SD}$.

${ }^{c}$ Obtained by counting the total number of tonic-style nerve terminals per muscle and dividing by the number of tonic muscle fibers ( $N=3$ muscles).

${ }^{d}$ Derived from electrophysiological data in Lichtman et al. (1985) $(N=50$ twitch muscle fibers, 57 tonic muscle fibers).

each segment a large percentage of the muscle fibers are in an alternating twitch, tonic arrangement. Because there are roughly equal numbers of twitch and tonic fibers, a certain degree of alternation would occur by chance. We found, however, that the alternation was significantly nonrandom (see Fig. 5 legend). For example, although there were instances in which two tonic or two twitch fibers were immediately adjacent, $96 \%$ of the fibers adjacent to tonic fibers in the preparation were of the twitch type. If fiber types were distributed randomly, this incidence would by only $55 \%$ (the percentage of twitch fibers in the muscle).

We also identified occasional muscle fibers associated with muscle spindles (intrafusal fibers). They typically had very small diameters and were recognizable by the presence of a short or long capsule (Fukami and Hunt, 1970). When an intrafusal fiber was present it seemed to substitute for a tonic fiber in the alternating pattern (Fig. 5).

There is evidence suggesting that the pattern of fiber types in this muscle is more elaborate than the simple twitch-tonic (or twitchintrafusal) alternation. While using the gold chloride staining procedure for nerve terminals (Boyd, 1962), we were surprised to observe differential staining of the muscle fibers themselves: some fiber types stained red, others blue, and some were unstained and appeared white. Furthermore, in many regions of the muscle the pattern of staining was red, white, blue, white, red, white, and so on (Fig. 6). By the criteria of terminal morphology and fiber diameter (Fig. 2, Table I), the unstained fibers (which appear white) were tonic. One class of twitch fibers (those having a rougher appearance and often of smaller diameter) stained red. A second class of twitch fibers (with a smooth appearance and often of larger diameter) stained blue. That the different staining characteristics of twitch fibers did in fact represent different subtypes of twitch fibers was confirmed by characterizing the tension properties of individual muscle fibers to single and tetanic stimuli (Fig. 7). One group of twitch fibers (blue-staining fibers with gold chloride) displayed a relatively fast rise-to-peak tetanic tension when stimulated intracellularly by a train of depolarizing current pulses (Fig. 7a). A second group of twitch fibers (red-staining fibers with gold chloride) more slowly reached peak tetanic tension but, unlike the faster contracting fibers, was more resistant to a decay in tension (Fig. $7 b$ ). These two twitch types appear similar to those described by Ridge (1971) in 

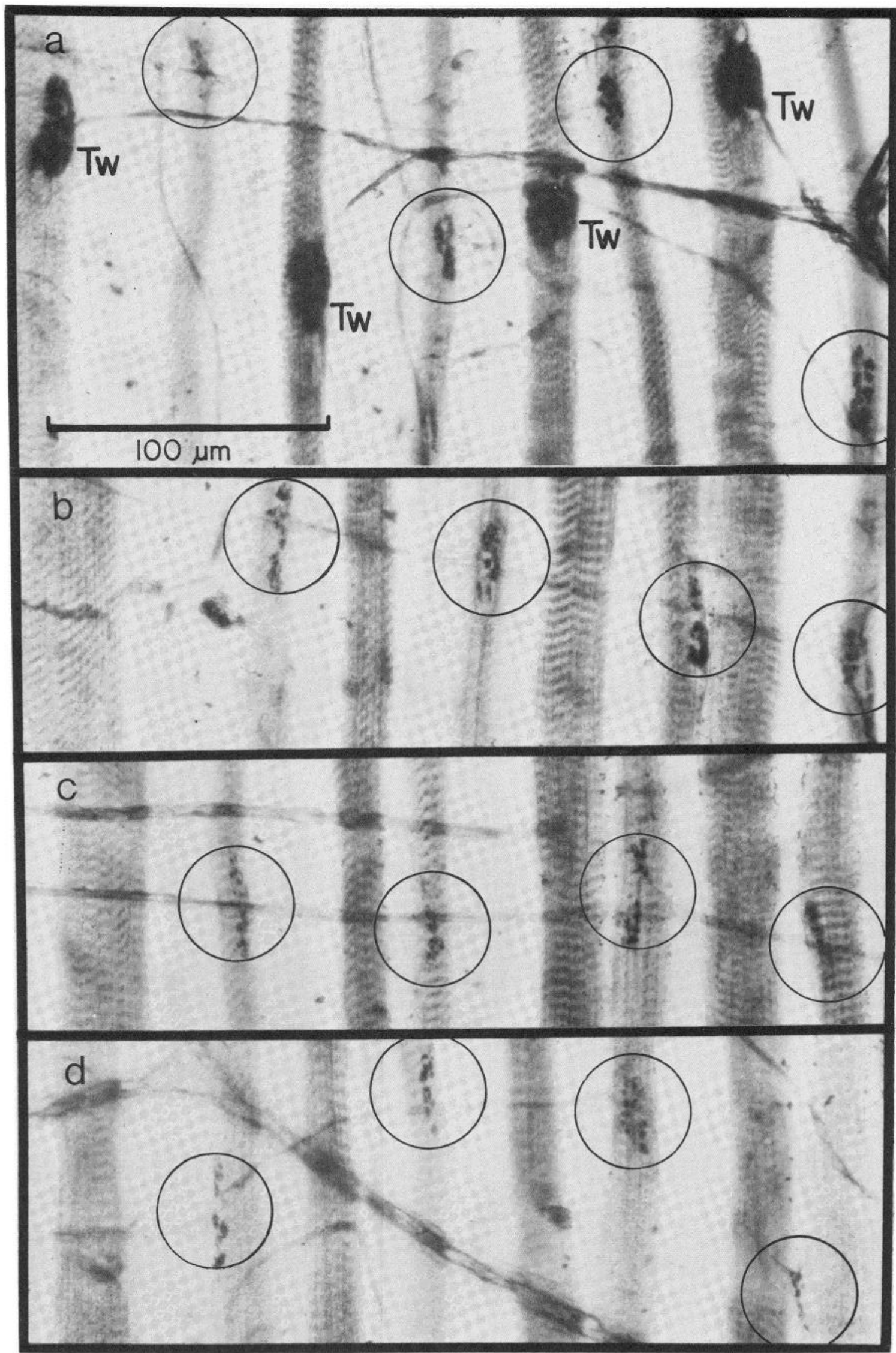

Figure 3. Endplate bands in the transversus abdominis stained with gold chloride. $a$, The main endplate band is located roughly midway along the length of the fibers and contains small (circles) and large ( $T w$ ) nerve terminals. The large nerve terminals are found only on singly innervated twitch fibers. Each of the smaller nerve terminals provides one of the approximately five to seven endplates to tonic muscle fibers distributed along their length. $b$ to $d$, Tonic endplate bands are spaced at regular intervals $(\sim 1 \mathrm{~mm})$ along the length of the muscle. In each of these panels the same muscle fibers are shown, but at tonic bands located approximately 1,2 , and $3 \mathrm{~mm}$ medial to the main endplate band shown in a. The small nerve terminals (circles) innervate only the tonic fibers in these bands. 


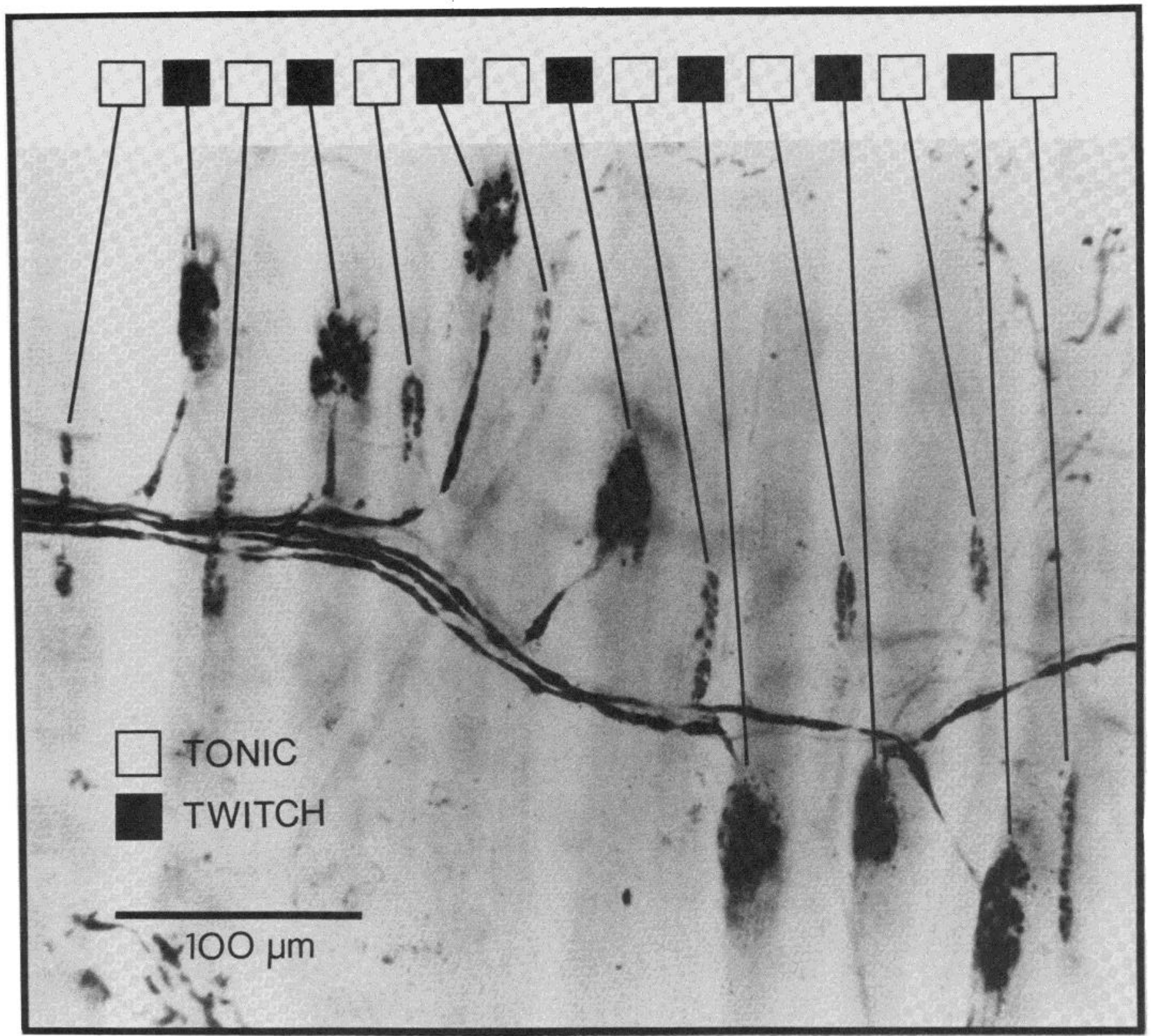

Figure 4. Sequence of fibers identified according to the criteria described in the text (gold chloride stain). Twitch fibers (large, solitary nerve terminals) are designated by solid squares and tonic fibers (small nerve terminals) are designated by open squares. In this area the small and large endplates strictly alternate. 
[-TONIC - IWITCH D-intrafusal

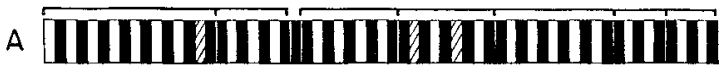

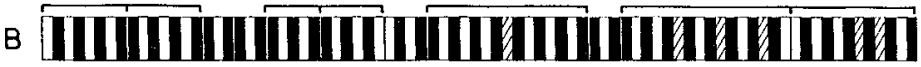

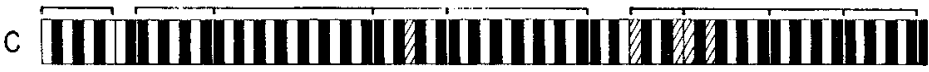

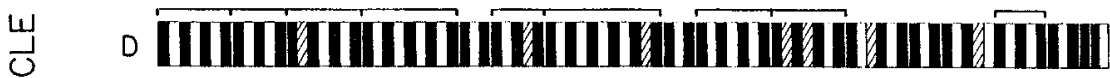

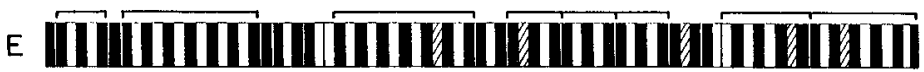

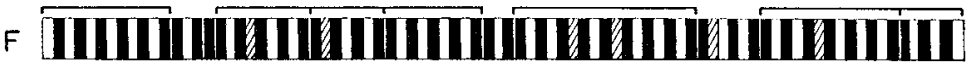

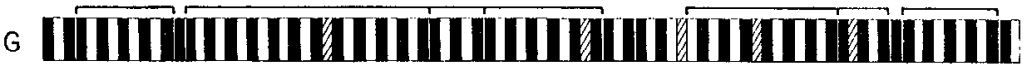

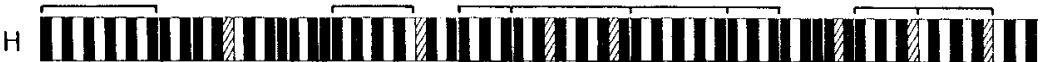

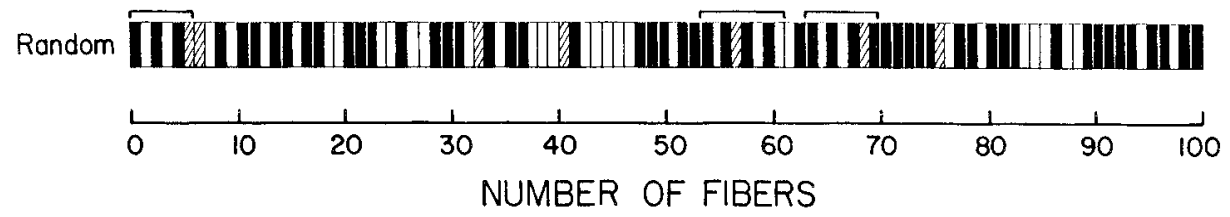

Figure 5. Diagram showing the arrangement of twitch (black) and tonic (white) muscle fiber types in each of eight contiguous segments $(A$ to $H$ ) of the transversus abdominis. Each vertical bar represents one fiber; the most caudal fiber is the leftmost bar in $A$; the most rostral is the rightmost bar in $H$. Intrafusal fibers associated with muscle spindles are shown as striped bars. The diameter of individual fibers is not represented. Classification was based on nerve terminal morphology, the prescnce of single or multiple innervation (Fig. 3), and, for intrafusal fibers, the presence of a sensory specialization surrounded by a short or long capsule. There were a total of $370(55 \%)$ twitch, $255(38 \%)$ tonic, and $44(7 \%)$ intrafusal fibers. The statistical significance of this distribution was analyzed by comparing the incidence of each type of fiber situated immediately adjacent (rostral) to a given fiber to each type's average incidence in the total population. Both the incidence of tonic next to twitch and that of twitch next to tonic were much higher than expected from a random distribution of fibers with the same percentages of types, whereas incidences of tonic next to tonic and twitch next to twitch were much lower; differences were all significant at the $p<0.0001$ confidence level (two-tailed). Interestingly, intrafusal fibers tended to substitute for tonic fibers within the pattern. Incidences of twitch next to intratusal and of intrafusal next to twitch were significantly higher than random, whereas those of tonic next to intrafusal and intrafusal next to tonic were significantly lower (all $p<0.01$ ). Shown at the bottom is a random sequence in which black ("twitch"), white ("tonic"), and striped ("intrasfusal") beads (in the same proportions as within the muscle itself) were drawn blindly from a beaker. There are many fewer regions of strict alternation in this sequence than in the muscle. Brackets above bars indicate regions where the alternation continues for at least five fibers in a row. 


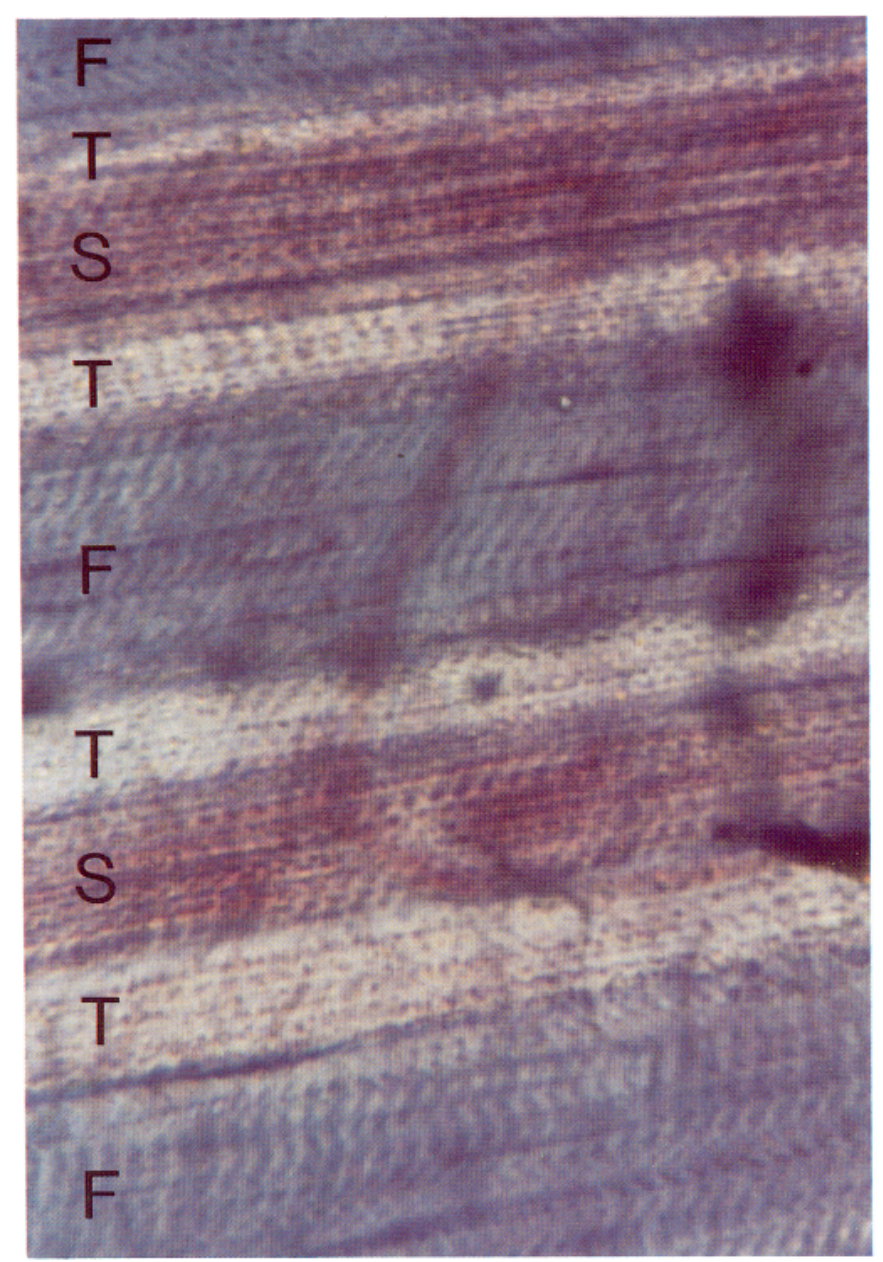

Figure 6. Repeating pattern of three fiber types in the snake transversus abdominis muscle. With gold chloride staining, slow-contracting twitch fibers $(S)$ are red, tonic fibers $(T)$ appear white, and fast-contracting twitch fibers $(F)$ are blue.

other snake muscles, except that we found no significant difference in their twitch times to peak (Fig. 7 legend). Much weaker and slower tensions are generated by tonic fibers (Fig. 7C). The fibers in Figures 6,7 , and 8 are labeled with the symbols $F$ (faster twitch), $S$ (slower twitch), and $T$ (tonic), according to the electrophysiological criteria shown in Figure 7. These designations, $\mathrm{F}$ and $\mathrm{S}$, are not to be confused with mammalian twitch fiber types. Indeed, preliminary results from microchemical enzymatic analysis indicate that the faster twitch fibers most closely resemble mammalian fast twitch fatigable fibers, whereas the slower twitch fibers correspond to mammalian fast twitch fatigue-resistant fibers (P. M. Nemeth, personal communication).

To see whether the secondary alternation of the two twitch fiber types (Fig. 6) is a general property of this muscle, we noted the gold chloride staining characteristics of each twitch muscle fiber in five contiguous muscle segments. Of the twitch fibers (256 of the 429 fibers in five segments), there were roughly equal numbers of the blue staining (52\%) and red staining (48\%) types. The regularity of red and blue twitch fiber alternation was computed by the same method used to analyze the twitch, tonic pattern (see Fig. 5 legend).

Both the incidence of a red staining fiber being the nearest twitch fiber to a blue fiber (69\%) and blue staining fiber being the nearest twitch fiber to a red fiber (76\%) were higher than expected from a random distribution of these two fiber types $(52 \%$ and $48 \%$, respec- tively), whereas the incidences of red nearest a red (24\%) and blue nearest a blue (31\%) were lower; differences were all significant at the $p<0.0001$ confidence level.

In accord with the morphological evidence for alternation, sequential recordings from contiguous groups of fibers showed an alternation of electrophysiological properties. Thus, fibers with twitch properties were usually immediately adjacent to fibers with tonic properties and, in addition, a secondary alternation among the twitch types was often discernible: twitch fibers which responded to intracellular stimulation with higher tetanic tensions and more rapid contraction times alternated with twitch fibers that were slower and weaker. Figure 8 shows a region of the muscle in which such physiological evidence for both a primary alternation of twitch and tonic fibers and a secondary alternation of two twitch fiber types was obtained.

\section{Discussion}

The transversus abdominis muscle is unusual in two respects. First, its fibers lie in a single-fiber-thick plane, in contrast to other muscles in which fibers occupy a three-dimensional volume. As a consequence, the arrangement of fiber types is a simple linear sequence (e.g., Fig. 5). Thus, any fiber type pattern, if it exists, is easy to detect and characterize.

The second unusual feature of the transversus abdominis, the regular pattern of fiber types, may be a consequence of the muscle's simplicity. Analogous cellular patterns in thick muscles, if present, might well be difficult to detect because of the greater geometric complexity.

One consequence of an alternating pattern is that fibers of the same type are rarely adjacent. Thus, muscle fibers within a motor unit in this muscle (which are strictly homogeneous in one of the three fiber types; R. S. Wilkinson and J. W. Lichtman, manuscript in preparation) are necessarily dispersed. Although no analogous pattern of fiber types has been observed in thick muscles, such anticlustering of fibers within motor units has been noted and proposed to have functional significance (Willison, 1980).

Although the fiber type pattern in this flat muscle may be functionally important, it may only be an epiphenomenon of muscle fiber differentiation. Whatever its role, the existence of such a pattern imposes constraints on the types of developmental strategies which could have been employed to generate fiber type diversity. Work in progress indicates that fibers innervated by individual axons within the muscle (motor units) are widely distributed, as occurs in other muscles. This rules out the possibility, however unlikely, that single axons acting indepedently might selectively innervate alternate muscle fibers in local regions of the muscle. But it is also difficult to conceive of motor axons of a particular type acting in concert to selectively innervate alternate myotubes from a homogeneous population. This would be required if neural influence alone were to establish the fiber type pattern. In contrast, if the location of twitch and tonic fibers were based on their lineage history such that, for example, myoblasts associated with neighboring primary myotubes fused to form secondary myotubes between them, the observed pattern might be a natural consequence. Lineage history is in fact related to the position of cells having a given phenotype in nematodes (Sulston and Horvitz, 1977) and in insect sensilla (Lawrence, 1966). In muscle, this could occur if separate groups of myotubes developed autonomously into the principal adult fiber types. Alternatively, the particular phenotype of a fiber may be instructed by its innervation. But in order to obtain a stereotyped alternating pattern, one would probably have to postulate that the respective maturation times of what are ultimately different fiber types must coincide with the times of ingrowth of different classes of motor axons. Thus, even if neural influence were to dictate properties of the individual fiber types, the fiber type pattern itself would remain an expression of the fibers' intrinsic developmental history. 
a

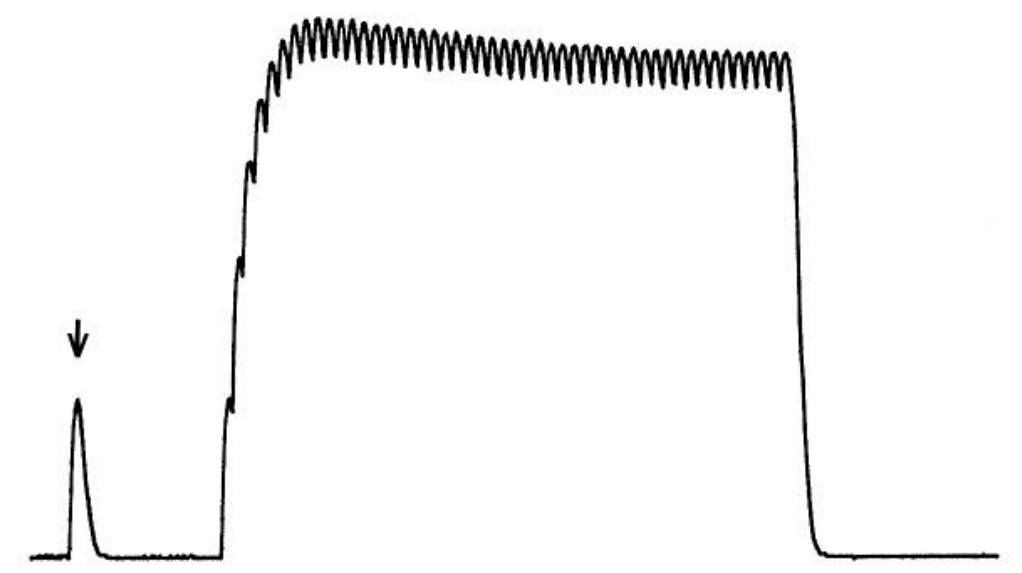

b

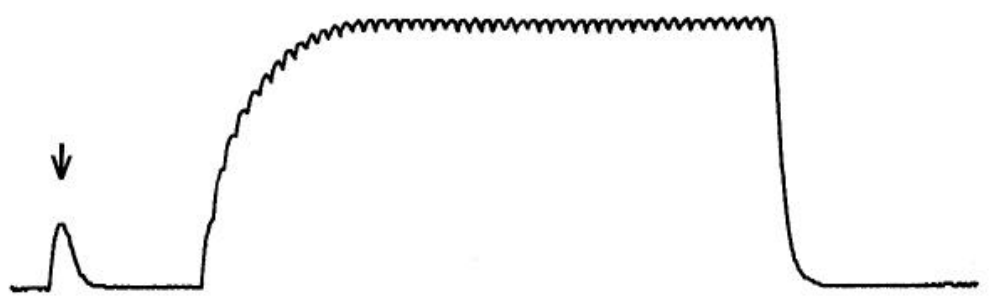

C
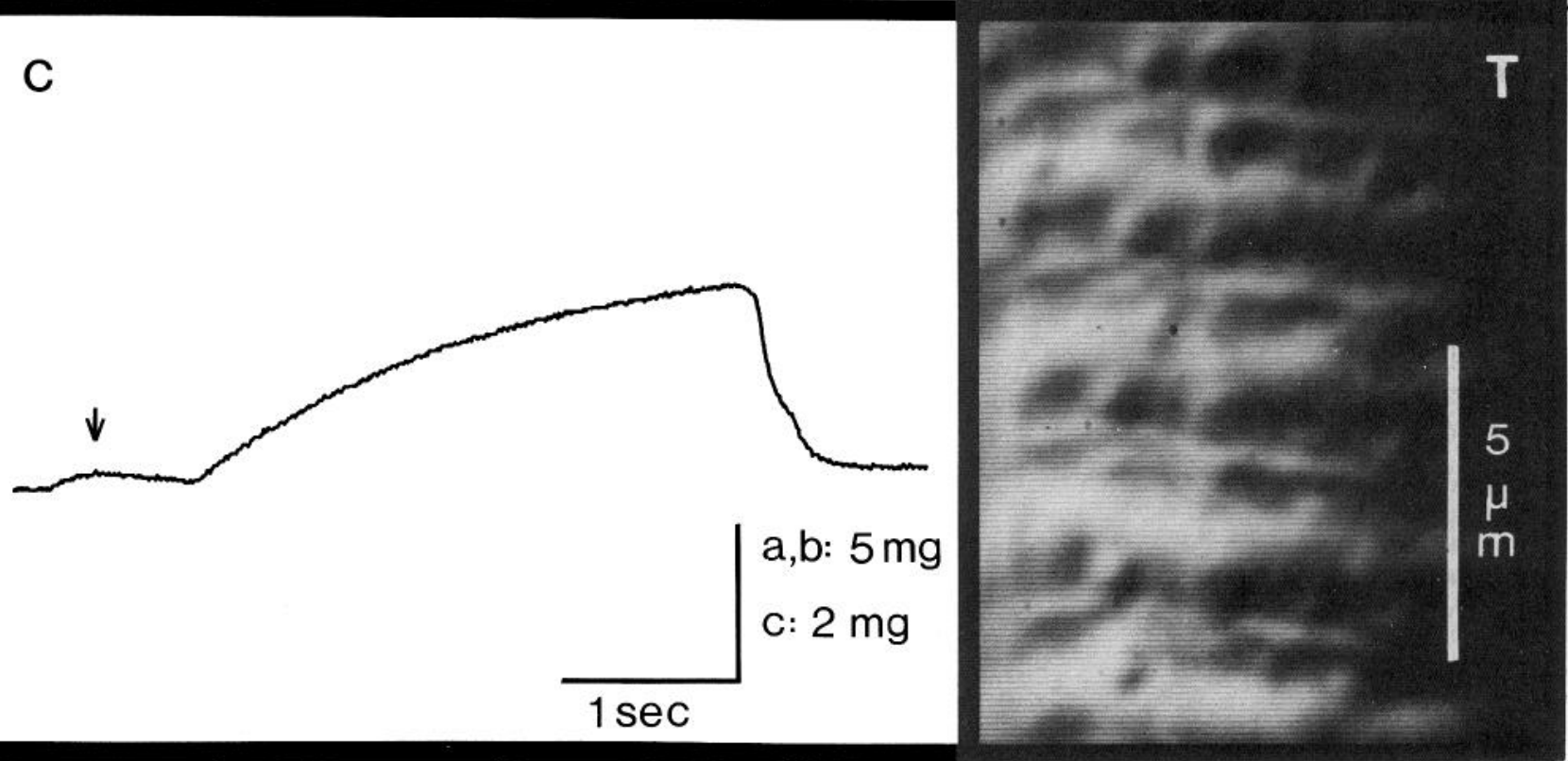


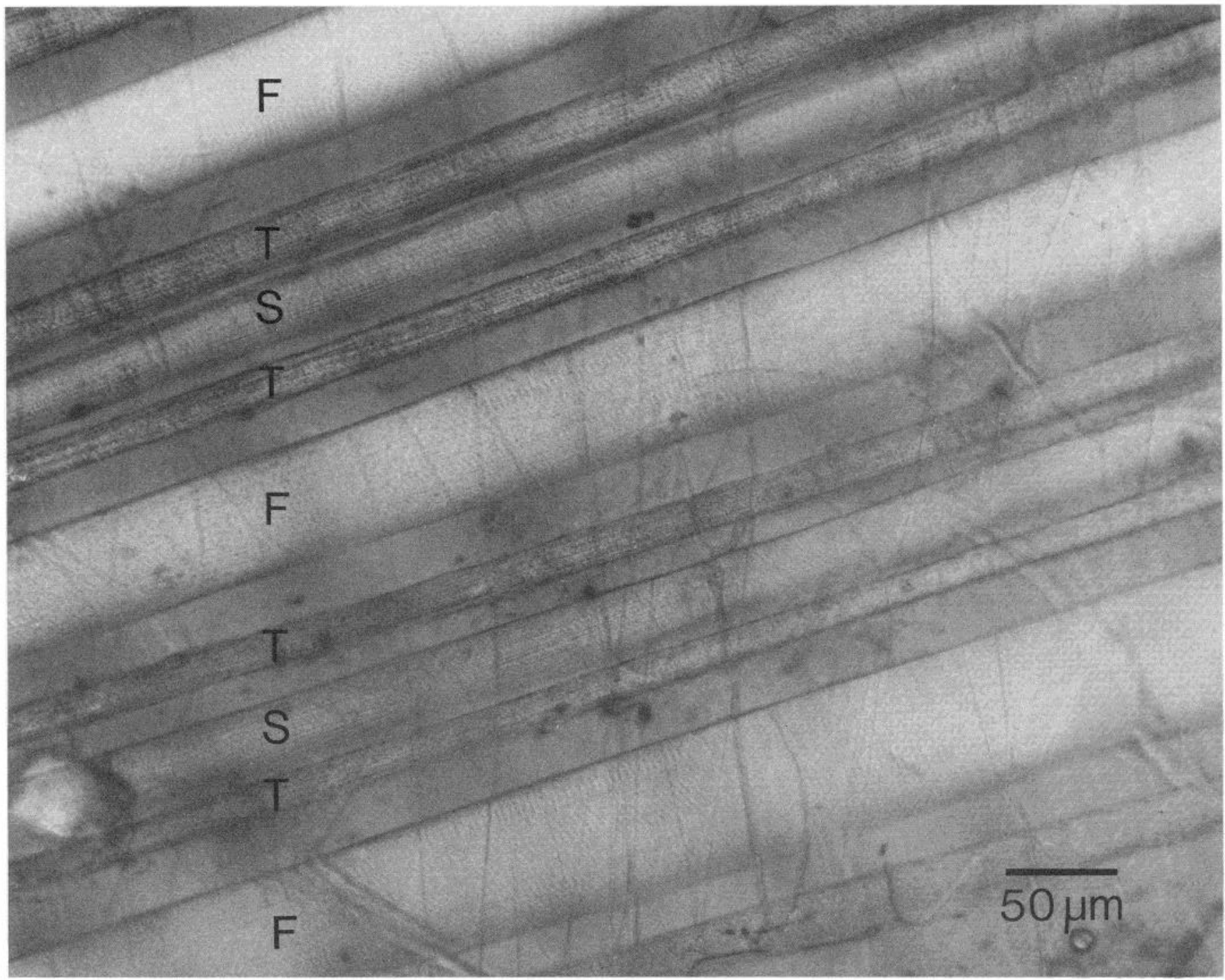

Figure 8. Nomarski photomicrographs of living electrophysiologically identified faster twitch $(F)$, slower twitch $(S)$, and tonic $(T)$ muscle fibers in the transversus abdominis. Note repeating pattern of fiber types $F, T, S, T$, and so on.

\section{References}

Askansas, V., S. Shafiq, and A. Milhorat (1972) Histochemistry of cultured aneural chick muscle. Morphological maturation without differentiation of fiber types. Exp. Neurol. 37: 218-230.

Boyd, I. A. (1962) Uniform staining of nerve endings in skeletal muscle with gold chloride. Stain Technol 37: 225-230.

Butler, J., E. Cosmos, and J. Brierley (1982) Differentiation of muscle fiber types in aneurogenic brachial muscles of the chick embryo. J. Exp. Zool. 224: $65-80$.
Cole, W. V. (1955) Motor endings in the striated muscle of vertebrates. J. Comp. Neurol. 102: 671-716.

Fukami, Y., and C. C. Hunt (1970) Structure of snake muscle spindles. J. Neurophysiol. 33: 9-27.

Hodgkin, A., and W. Rushton (1946) The electrical constants of a crustacean nerve fibre. Proc. R. Soc. Lond. (Biol.) 133: 449-479.

Karpati, G., and W. K. Engel (1968) "Type grouping" in skeletal muscles after experimental reinnervation. Neurology 18: 447-455.

Kelly, A. M. (1983) Emergence of specialization in skeletal muscle. In Handbook of Physiology. Sect. 10: Skeletal Muscle, L. D. Peachey, ed., pp. 507-538, Williams and Wilkins, Baltimore.

Figure 7. Tension responses (left) and surface appearance using Nomarski optics (right) of three principal fiber types in the transversus abdominis Tension responses to a single current pulse $(2 \mathrm{msec}, 100 \mathrm{nA})$ delivered intracellularly produced a measurable contraction in each muscle fiber type (arrows) The amplitude of the twitch was largest for the smooth-appearing twitch fibers (a), intermediate for the rough-appearing twitch fibers (b)., and weakest for the tonic fibers $(\mathrm{c})$. The time to peak of these single twitches was significantly shorter for the two twitch fiber types (smooth: $49 \pm 11$ msec, mean \pm SD, $N$ = 10; and rough: $53 \pm 7 \mathrm{msec}, N=10$ ) than for the tonic fibers (>200 msec) using the same stimulus protocol. When the current pulses were delivered as a train $(16.6 \mathrm{~Hz}$ for $3 \mathrm{sec})$ they produced a tetanic contraction which differed in each fiber type. As shown in the upper right panel, the smooth-appearing fibers reached maximum tension fastest $(80 \%$ maximum tension, $0.21 \pm 0.05 \mathrm{sec}, N=10)$, but the tension usually decayed slightly over a period of seconds. These fibers are referred to as faster $(F)$ in the text. The rougher-appearing twitch fibers (middle right panel) reached peak tetanic tension more slowly $(0.47 \pm 0.09 \mathrm{sec}, N=10)$ and are referred to as slower $(S)$. Once reached, their tension remained high. Tonic ( $T)$ fibers (lower right panel) were extremely slow to reach peak tension ( $>2 \mathrm{sec})$. 
Kuffler, S., and D. Yoshikami (1975) The distribution of acetyicholine sensitivity at the post-synaptic membrane of vertebrate skeletal twitch muscles. J. Physiol. (Lond.) 244: 703-730.

Lawrence, P. (1966) Development and determination of hairs and bristles in the milkweed bug, Oncopeltus fasciatus (Lygaeidae, Hemiptera). J. Cell Sci. 1: $475-498$.

Lichtman, J. W., R. S. Wikinson, and M. M. Mark (1985) Multiple innervation of tonic endplates revealed by activity-dependent uptake of fluorescent probes. Nature 324: 357-359.

Nougues, J., and F. Bacou (1977) Enzymatic activities of muscle fibres differentiated, in vitro, from pectoralis major (white) and adductor magnus (red) muscles of chick embryos. Experientia 33: 714-716.
Ridge, R. M. A. P. (1971) Different types of extrafusal muscle fibres in snake costocutaneous muscles. J. Physiol. (Lond.) 217: 393-418.

Rubinstein. N., and A. Kelly (1978) Myogenic and neurogenic contributions to the development of fast and slow twitch muscles in the rat. Dev. Biol. 62: $473-485$.

Sulston, J., and A. Horvitz (1977) Post-embryonic cell lineages of the nematode, Caenorhabditis elegans. Dev. Biol. 56: 110-156.

Thompson, W. L. Sutton, and D. Riley (1984) Fibre type composition of single motor units during synapse elimination in neonatal rat soleus muscle. Nature 309: 709-711.

Willison, R. G. (1980) Arrangement of muscle fibers of a single motor unit in mammalian muscles. Muscle Nerve 3: '360-361. 